\title{
Pure Electric Bus Crashworthiness Analysis
}

\author{
Wen-wei Wang \\ National Engineering Laboratory for \\ Electric Vehicles \\ Beijing Institute of Technology \\ Beijing 100081, China \\ bitev@bit.edu.cn
}

\author{
Cheng-jun Zhou \\ National Engineering Laboratory for \\ Electric Vehicles \\ Beijing Institute of Technology \\ Beijing 100081, China \\ zcj87@163.com
}

\author{
Jiao-yang Chen \\ National Engineering Laboratory for \\ Electric Vehicles \\ Beijing Institute of Technology \\ Beijing 100081, China \\ joyoung3@sina.com
}

\begin{abstract}
This paper has analyzed the basic theory and material constitutive relation of crashworthiness simulation. The finite element model for vehicle side crash has been built and the side crash simulation with an initial velocity of $50 \mathrm{~km} / \mathrm{h}$ has been conducted. Though the structure deformations analysis of each part, the side crash impacts on passenger living space and battery have been predicted and the crashworthiness of the pure electric bus body structure has been tested.
\end{abstract}

Keywords-pure electric bus; crashworthiness; side crash

\section{INTRODUCTION}

The development of new-energy vehicles is becoming one of the most significant ways in transportation to solve the world wide problems of energy, environment and climate change. As a new-energy vehicle, pure electric vehicle has obvious advantages on environment protection and energy saving, compared with traditional combustion vehicles. However, due to the battery and other electric systems, the safety of electric vehicles should be carefully deliberated. The battery safety is the crucial point, because of the danger caused by crash, such as high-voltage current, electrolyte leakage and even battery system explosion. Thus, it is of great significance to simulate the electric bus battery tank crash, in order to improve its crashworthiness.

\section{BASIC THEORY OF VEHICLE CRASH ANALYSIS}

\section{A. The Simulation Algorithm}

The vehicle crash simulation process is essentially to solve a partial differential equation with given initial and boundary conditions. Specially, the dynamic contact boundary conditions are unknown before solving the problem, namely, it is a typical dynamic contact problem ${ }^{[1]}$. The crash process has strongly nonlinear features and is transient, thus generally explicit simulation algorithm, namely central difference method has been used, which diagonalizes mass matrix based on virtual work principle and solves the second order differential system.

\section{B. Material Constitutive Relation}

Material constitutive relation refers to the variation relation between stress and strain or strain rate. It is a key factor to influence the crash simulation accuracy. In the crash process, there exist various plastic-deformation-based energy absorption methods. Thus, to select a reasonable elastic-plastic model to reflect the material stress-strain relation correctly is very important to the crash analysis accuracy.

The software Ls-dyna provides various material elasticplastic math models. All the steel parts of the electric bus, in this paper, have been expressed as segmented plastic material model $^{[2]}$. Besides, because battery is a complex of multiple materials, including copper foil, aluminum foil, cathode and anode material, electrolyte and plastic or steel shell, this paper based on the battery material structure and material characteristics, has chosen isotropic plastic model to present battery.

\section{CRASH FINITE ELEMENT MODEL BUILDING}

\section{A. Vehicle Side Crash Finite Element Model}

After the pure electric bus body frame model has been built in CATIA, import it into HyperMesh. After the geometry cleanup and simplifying the structure, the model meshing begins. The bus frame is constituted all by thin-wall square fittings, thus choose "shell" as model element type. While choosing element size, due to crash local effect, choose a precise size of $20 \mathrm{~mm}$ in collision area and a size of $50 \mathrm{~mm}$ in non-collision area in order to reduce calculation scale and improve working efficiency.

About material: choose elastic material (MAT1) as the materials for body front panel, rear panel and left side panel, and choose nonlinear material (MAT24) for right side panel, floor and roof. Because the wheels are not in collision area during the crash, set them as rigid. When consider the battery system, set cell box as nonlinear material (MAT24), set battery rack as elastic material (MAT1) with a density of $1.95 \mathrm{e} 3 \mathrm{~kg} / \mathrm{m} 3$. Because the materials of battery outer packing and electrode current conductor, copper foil and aluminum foil, are easily plastically deformed, choose power index plastic material for battery and set cathode material density of $3.3 \mathrm{e} 3 \mathrm{~kg} / \mathrm{m} 3$ as battery density.

About connection: take node coupling method for T-shaped connections between the rods and use "Spotweld" method offered in HyperMesh to simulate other connections, mainly including the connections between roof, front and rear panel, left and right panel, and floor, etc. 
The total node number in the finite element model is 198253, and the element number is 173267 . The finite element model is shown in Figure 1.

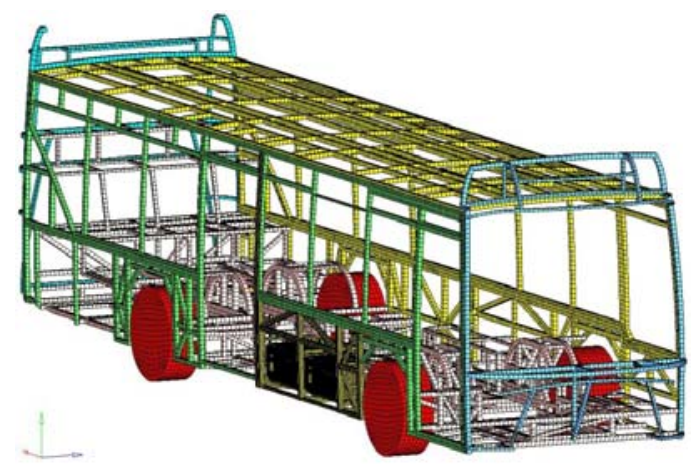

Figure 1. The finite element model for electric bus

\section{B. Finite Element Model of Mobile Deformable Barrier}

The mobile deformable barrier consists of sled and energy absorber. The stiffness of front energy absorber represents the average front stiffness of the local vehicles. The dimension of mobile deformable barrier, the ground clearance of absorber, and the mass and mass center position all meet the requirements of Regulation GB20071-2006, "Passenger Protection of Vehicle Side Crash" ${ }^{\text {[3] }}$ :

- The total mass should be $950 \pm 20 \mathrm{~kg}$; the front and rear tracks of sled should be $1500 \pm 10 \mathrm{~mm}$; the wheel base should be $3000 \pm 10 \mathrm{~mm}$; the center of gravity should be off the longitudinal symmetrical plane within $10 \mathrm{~mm}$, and be $1000 \pm 30 \mathrm{~mm}$ off the front axle, $500 \pm 30 \mathrm{~mm}$ off the ground.

- The distance between barrier front surface and gravity center should be $500 \pm 30 \mathrm{~mm}$; the width and height of barrier deformation area should be $1500 \pm 10 \mathrm{~mm}$ and $500 \pm 5 \mathrm{~mm}$; the static ground clearance before crash should be $30 \pm 3 \mathrm{~mm}$.

Generally use aluminum honeycomb as deformation energy absorber, which can simulate the vehicle front deformation stiffness. The absorber can be divided into 6 independent parts with 2 layers and there would be 3 parts for each layer. All the parts have the same width of $500 \pm 5 \mathrm{~mm}$ and the same height of $250 \pm 3 \mathrm{~mm}$, except thickness: the upper layer is $440 \pm 5 \mathrm{~mm}$ and the lower is $500 \pm 5 \mathrm{~mm}$. The thicker lower layer is to simulate the bumper bulge. The detailed structure is shown in Figure 2.

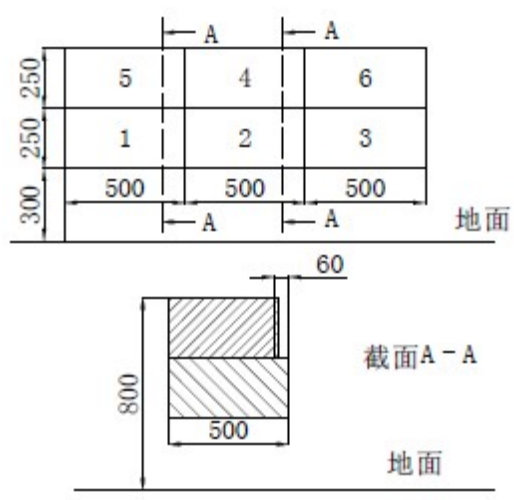

Figure 2. Energy absorber dimensions and part positions

In the finite element model of mobile deformable barrier, choose shell elements to present the sled and energy absorber mounting plate, and choose hexahedron elements for absorber and wheels. Set aluminum honeycomb (MAT26) as the material for absorber, and isotropy linear elastic material (MAT1) for body frame, wheels and honeycomb mounting plate. Select node coupling method to connect wheels, sled frame and absorber mounting plate, and select "Spotweld" to connect absorber and mounting plate.

The finite element model of mobile deformable barrier has been built as shown in Figure 3.

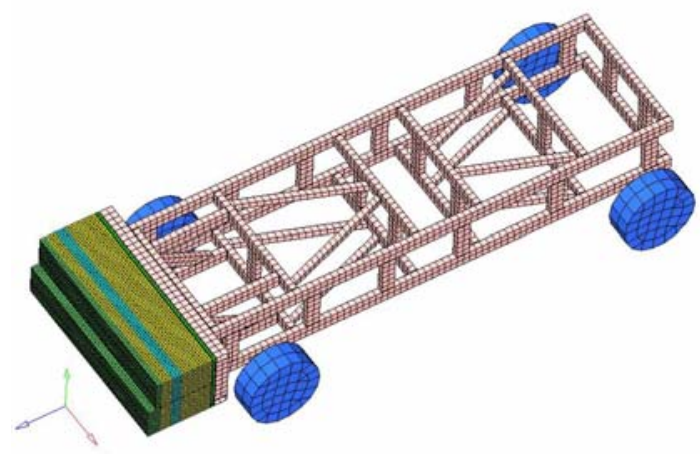

Figure 3. The finite element model for the mobile deformable barrier

\section{RESULT ANALYSIS OF SIDE CRASH SIMULATION}

\section{A. Parameter Defination for Crash Simulation}

1) The ground modeling: Import the mobile deformable barrier into vehicle model and adjust its place to make the wheel lowest point of bus and barrier in the same level. During the modeling of vehicle side crash simulation, a rigid wall needs to be built with a horizontal distance of $1 \mathrm{~mm}$ lower from the wheel lowest level. Using the "rigid walls" menu in HyperMesh can the rigid wall be built.

2) Initial velocity setup: In the analysis of pure electric bus side crash process, setting initial velocity of the barrier is essential. According to the crash regulations, use the key word “*INTIAL VELOCITY” to give all nodes in the barrier finite 
element model a initial velocity of $50 \mathrm{~km} / \mathrm{h}$, with a direction of horizontally left.

3) Gravity acceleration setup: The process of vehicle side crash should take the influence of gravity acceleration into consideration. Apply a vertical downward acceleration of $9.8 \mathrm{~m} / \mathrm{s} 2$ to both the finite element models of bus and barrier.

4) Contact defination: Use "interfaces" to define the crash contact. Define the constraint type in side crash as "CONTACT_AUTOMATIC_SINGLE_SURFACE" and the contact stiffness SLSFAC as 0.1. Set static and kinetic friction coefficients between each part, FS and FD as 0.2.

5) Box setup: In side crash, because the bus body is very long, comparing with collision area parts, the parts far away from the collision area have small deformations, or even none contact. In order to improve the calculate efficiency, define a box in the evaluated crash area. In this wise, the contact problem is only searched inside the area of box, and the area out of the box would not be taken into consideration.

6) Hourglass control: Hourglass mode is a nonphysical zero-energy deformation mode, which produces zero stress and strain. The single point integration method in Ls-dyna solver will lead to an element deformation without any energy consumption, namely hourglass mode. In this paper, select viscous hourglass control method to control hourglass mode. Set the control coefficient as 0.05 , which means the hourglass energy must not exceed $5 \%$ of system total internal energy, to improve the reliability of the calculation results.

7) Solving time defination: Generally in crash test, the crash process of sedan would come to the end within $120 \mathrm{~ms}$ after contacting the rigid wall. Because the bus is much heavier than a sedan, the process of bus crash is much longer than that of sedan. In this paper, set the crash duration as 180ms.

\section{B. Deformation Analysis of Vehicle Body Frame}

The crash result analysis indicates that, during the $180 \mathrm{~ms}$ side crash, the crash process can be divided into 4 stages according to the displacement features of each frame part. The displacement changing of body crucial parts are shown in Figure 4 and the vehicle body displacement cloud pictures along with timeline are shown in Figure 4-6.

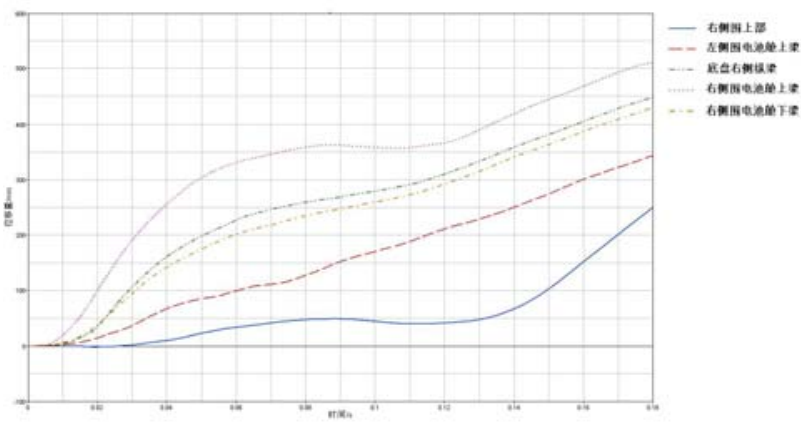

Figure 4. The displacement changing of body crucial parts

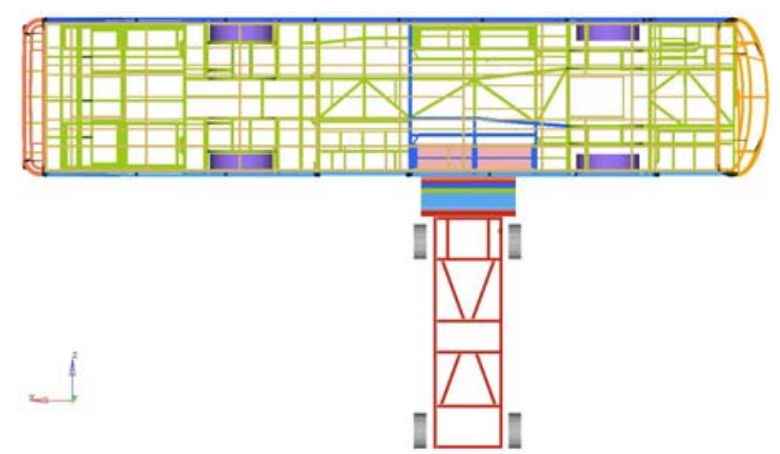

Figure 5. At $\mathrm{t}=0 \mathrm{~s}$, the vehicle body displacement cloud pictures

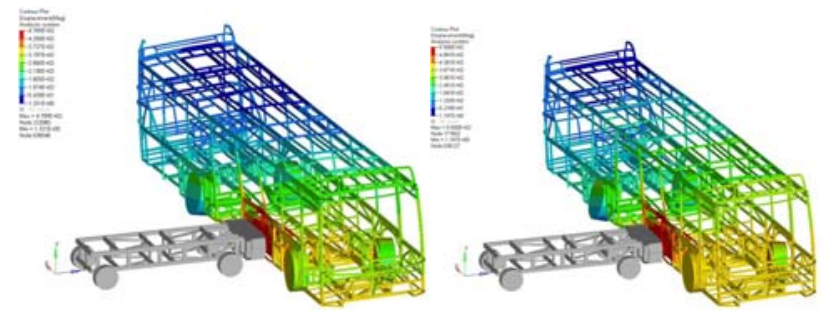

Figure 6. The vehicle displacement cloud pictures at $t=150 \mathrm{~s}$ and $\mathrm{t}=180 \mathrm{~s}$

1) During $0-20 \mathrm{~ms}$, because the mobile deformable barrier firstly contacts the battery tank skin, bending deformations of skin structure and tank door have taken place. The deformation is small, but the deformation velocity becomes gradually larger. After $20 \mathrm{~ms}$, the battery tank frame starts to deform and at this moment the biggest displacement of vehicle body is $138 \mathrm{~mm}$. The contact points of four wheels with the ground have not changed, which means during 0$20 \mathrm{~ms}$ the vehicle did not move relative to the ground.

2) During $20-100 \mathrm{~ms}$, firstly the battery tank frame and some rods of vehicle right panel have deformed to absorb energy and to cushion the bus frame deformation. Along with the collision, the material rigidfication of battery tank frame and tank door frame has occurred, which means the deformation is basically complished. The longitudinal and cross beams on the right of chassis are becoming deformed. After 40ms, the crash energy has been delivered to the left longitudinal and afterwards the left cross beam and the left panel. The left panel becomes deformed. After $50 \mathrm{~ms}$, the bus body biggest displacement is $348 \mathrm{~mm}$ and it reaches $415 \mathrm{~mm}$ after $100 \mathrm{~ms}$. From $20 \mathrm{~ms}$, the front wheels begin to slip relatively to the ground and the rear wheel slip occurs from about $25 \mathrm{~ms}$.

3) During 100-150ms, obvious horizontal anticlockwise rotation of bus body has occurred. On one hand, some of the rods which are still of elasic deformation, can get some deformation recovery during this process, which reduces the total deformation of the bus frame. But on the other hand, due to the crash inertia force, the displacement of bus body is keeping increasing. At $150 \mathrm{~ms}$, the body displacement reaches the periodic maxmum $479 \mathrm{~mm}$.

4) During $150-180 \mathrm{~ms}$, the bus slips approaching to the crash direction. Meanwhile, bus body begins to separate from the mobile deformable barrier. During this process, the rods 
still of elastic deformation get recovery and the most of the rods do not deform any more. At $180 \mathrm{~ms}$, all the rods recover from the deformation and the displace of the electric bus reaches the maxmum $550 \mathrm{~mm}$. The crash process ends.

The width of the electric bus body is $2540 \mathrm{~mm}$ and the width between the seats above left and right battery tanks is $1150 \mathrm{~mm}$. The maxmum passenger living space intrusion introduced by battery tank is $158 \mathrm{~mm}$ which indicates that, the side crash has small influence on passenger living space. However, considering the seats location, the battery tank and right panel deformation may do harm to passenger's waist and legs. Because the crash has occurred in the middle of the bus body, the impact to front and rear panels is not obvious and these panels only move along with the bus.

In the vehicle side crash process, thanks to the cushioning effect of battery tank door and the bus frame, the battery system has not been collided directly, which reduces the harm to battery and protects the battery from squashing and danger. However, because the fast melting insurance is placed in the front of the battery, when crash occurred, the tank door would touch the insurance and might get it damaged or short out, and lead to battery fire. The follow-up assignments of the paper are to optimize the structures of battery tank door and tank frame, to leave a safe distance for the battery tank and to improve the safety of battery system.

\section{CONCLUSION}

In this paper, the vehicle crash simulation theory has been analyzed and the electric bus' and mobile deformable barrier's finite element models have been built. With the help of Lsdyna solver, the vehicle side crash simulation and deformation analysis have been conducted. Through the discuss of the impact caused by side crash on passenger living space and battery system, the frame structure crashworthiness has been confirmed. In the end, the frame structure improvement suggestions have been offered.

\section{REFERENCES}

[1] Zhang Juehui, Tan Dunsong and Gao Weimin, "The FEM for vehicle crash and doors crashworthiness study,” Journal of Tongji University, vol.25, pp. 450-453, April 1997.

[2] Song D, Mack P and Tarriere C, "Finite element simulation of the occupant's belt interaction: chest and pelvis deformation,” Belt Sliding and Submarining.

[3] Shi Shengjie, "Side crashworthiness analysis of pure electric bus," Beijing: China machine press, 2010. 